\title{
Resveratrol is cytotoxic and acts synergistically with NF- $\kappa$ B inhibition in osteosarcoma MG-63 cells
}

\author{
Ning $\mathrm{Xu}^{1}$, Lili Wang ${ }^{2}$, Shiping $\mathrm{Fu}^{1}$, Bin Jiang ${ }^{2}$
}

\begin{abstract}
${ }^{1}$ Department of Orthopedics, Shanghai Eighth People's Hospital, Shanghai, China 2Department of Oncology, Ninth People's Hospital Affiliated to Medical College of Shanghai Jiaotong University, Shanghai, China
\end{abstract}

Submitted: 28 August 2017

Accepted: 9 January 2018

Arch Med Sci 2021; 17 (1): 166-176

DOI: https://doi.org/10.5114/aoms.2020.100777

Copyright @ 2020 Termedia \& Banach

\section{Abstract}

Introduction: Osteosarcoma is the most common primary malignancy of the bone. The existing adjuvant chemotherapy regimens, while improving the overall survival, have been limited by the significant systemic toxicity. Substantial clinical and research efforts are being invested to develop novel pharmaceutical agents. Resveratrol (Res) has been suggested to have a chemopreventive effect. However, the mechanism of Res in osteosarcoma remains to be elucidated.

Material and methods: The MG-63 osteosarcoma cell culture model was used to investigate the chemotherapeutic effect of Res. MTT assay, wound healing assay, and Transwell migration assay were used to document the effect of Res on cell proliferation, migration, and invasion, respectively. Apoptosis in MG-63 cells was quantified with the TUNEL assay. Western blotting analysis was used to examine the molecular changes following Res treatment. Data processing and analysis were conducted using GraphPad Prism 5.0. $P<0.05$ was considered statistically significant.

Results: Our data suggested that Res blocks cell proliferation, migration, and invasion, and activates apoptotic cell death in osteosarcoma MG-63 cells.

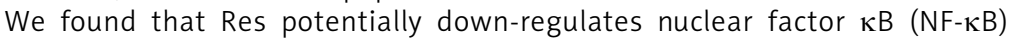
and Akt intracellular signaling transduction. Moreover, the combination of Res and pyrrolidine dithiocarbamate (PDTC), an NF- $\kappa B$ inhibitor, resulted in synergistic growth inhibition of osteosarcoma.

Conclusions: Our in vitro preclinical study in the MG-63 cell line model supports the translation of Res to the clinical management of patients with osteosarcoma.

Key words: proliferation, migration, invasion, apoptosis, preclinical, translational, MG-63, chemotherapy, signal transduction.

\section{Introduction}

Osteosarcoma is the most common primary malignancy of the bone, occurring predominantly in adolescents and children. Data from 19732004 suggested that the incidence of osteosarcoma was approximately 4.4 cases per 1 million people who were aged 24 years or younger [1]. Osteosarcoma was treated primarily with surgical resection (amputation or limb-salvage surgery). With the adjuvant treatment with pre-op and/ or post-op chemotherapy, the survival of osteosarcoma has been remarkably improved over the past several decades [2, 3]. One study showed that the 5-year survival in osteosarcoma patients under 15 years was

\author{
Corresponding author: \\ Bin Jiang \\ Department of Oncology \\ Ninth People's Hospital \\ Affiliated to Medical College \\ of Shanghai Jiaotong \\ University \\ No. 280, Mohe Road \\ Baoshan District \\ Shanghai, 201901, China \\ Phone: +86 21-56691249 \\ Fax: +86 21-56691663 \\ E-mail: drbinjiang@outlook. \\ com
}


increased from $40 \%$ to $76 \%$, and in adolescent patients from 56\% to $66 \%$ between 1975 and 2010 [4]. Nevertheless, the existing regimens including combinational use of high dose methotrexate, doxorubicin, cisplatin, epirubicin, ifosfamide, cyclophosphamide, etoposide, gemcitabine and topotecan have been limited by the significant systemic side effects such as hepatic and neural toxicity. Further, a subset of patients responded poorly to the established chemotherapy or relapsed after therapy [5]. Their prognosis has shown little or no improvement over the past 20 years. As such, considerable efforts have been actively spent to develop novel agents that can be used alone, or in combination with current standards of care, for the management of osteosarcoma.

Resveratrol (Res) is a polyphenolic phytoalexin natural compound obtained from various fruits and red wine. Initially recognized for its anti-inflammatory, anti-angiogenic and anti-oxidative effects, Res has been widely studied for the antitumor activities in various cancer models [6-9] since the first report in a mouse skin cancer model [10]. Significant evidence has suggested that Res has chemotherapeutic or chemopreventive effects on several cancer types [11-13]. For example, Res suppressed the growth and metastasis of SW480 human colon cancer cells via the induction of microRNA 663 [14]. A study by Zulueta et al. additionally showed that Res altered intracellular signaling necessary for cell growth arrest and apoptosis in gastric carcinoma [15].

Given the existing evidence, it is imperative to investigate the efficacy of Res in osteosarcoma.

There have been a number of relevant studies available from the public databases. Initially, Zou et al. screened 14 botanical extracts and found that Res had significant anti-osteosarcoma activities in blocking cell proliferation and activating apoptosis [16]. These events were associated with the inhibition of canonical Wnt/ $\beta$-catenin signaling. Further, Res inhibited HIF-1 $\alpha$ protein expression and hypoxia-enhanced proliferation, invasion, and epithelial to mesenchymal transition in osteosarcoma [17]. Li et al. found that Res induced apoptosis in four osteosarcoma cell lines: HOS, Saos-2, U2-OS and MG-63 [18]. The authors postulated that the effects of Res were mediated through silent information regulator 2 (Sirt1), given that a similar effect was elicited by a Sirt1 activator, isonicotinamide, and that resveratrol was enhanced by nutrition restriction by L-asparaginase. An study by Liu et al. likewise investigated the cell apoptosis in MG-63 cells by resveratrol, wherein the authors observed that Res could inhibit growth and induce apoptosis [19]. Preliminary observational data suggested the molecular mechanism related to the modulation of survivin and p21. Finally, Res had an inhibitory effect on vascular endothelial growth factor expression and U2-OS cell proliferation [20]. Thus, it appeared generally accepted that Res induces cytotoxicity in osteosarcoma, yet possibly through diverse molecular mechanisms and intracellular processes. Significant further work is also needed to attract the clinical use of Res in oncology, as opposed to the dietary use from its wellknown cardiovascular protective activity.

In this study, we used the MG-63 osteosarcoma cell culture model and assessed the chemotherapeutic effect of Res. In line with earlier publications, our results suggested that Res blocks cell proliferation, migration, and invasion, and activates apoptotic cell death in osteosarcoma. Notably, we instead found that Res potentially down-regulates nuclear factor $\kappa \mathrm{B}(\mathrm{NF}-\kappa \mathrm{B})$ and Akt intracellular signaling cascades. Res, when combined with an NF$\kappa \mathrm{B}$ inhibitor, pyrrolidine dithiocarbamate (PDTC), results in synergistic and pronounced growth inhibition of osteosarcoma. Together, our in vitro preclinical study is among the first to report the synergistic action of Res and PDTC, and supports the translation of Res (alone or in conjunction

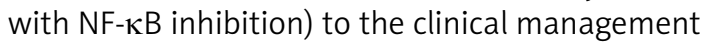
of patients with osteosarcoma.

\section{Material and methods}

\section{Materials}

Resveratrol and pyrrolidine dithiocarbamate were purchased from Sigma-Aldrich (St Louis, MO, USA). Res was dissolved in dimethyl sulfoxide (DMSO) and PDTC was dissolved in phosphate-buffered saline (PBS). Both reagents were stored at $-80^{\circ} \mathrm{C}$. Tetrazolium dye MTT and sodium pyruvate were acquired from Aladdin (Shanghai, China). Matrigel basement membrane matrix was purchased from BD Biosciences (NJ, USA).

\section{Cell culture}

The human osteosarcoma cell line MG-63 was obtained from iCell (Shanghai, China). MG-63 cells were maintained in the Minimum Essential Media (MEM) culture medium (Gibco, MA, USA), supplemented with $10 \%$ fetal bovine serum (FBS, Gibco, MA, USA), $100 \mathrm{kU} / \mathrm{l}$ penicillin and $100 \mathrm{mg} / \mathrm{l}$ chloramphenicol (Gibco, MA, USA) and $0.11 \mathrm{~g} / \mathrm{l}$ sodium pyruvate at $37^{\circ} \mathrm{C}$ with $5 \%$ carbon dioxide $\left(\mathrm{CO}_{2}\right)$. Cells are routinely passaged with $0.25 \%$ trypsin digestion. Changes in cell morphology and growth were monitored with an inverted optical microscope.

\section{MTT assay}

MG-63 cell proliferation was determined by the tetrazolium dye 3-(4,5-dimethyl-2-thiazyl)-2,5-diphenyl-2H-tetrazolium bromide (MTT) assay. Brief- 
ly, $5 \times 10^{3}$ MG-63 cells $(100 \mu \mathrm{l})$ in the exponential phase of growth were plated in 96-well culture plates. After $24 \mathrm{~h}$, cells were divided into three groups: group one was treated with $\operatorname{Res}(0,25$, $50,100$ or $200 \mu \mathrm{M})$, group two was treated with $\operatorname{PDTC}(0,3,10,30,50$ or $100 \mu \mathrm{M})$, and group three was treated with the combination of Res (fixed at $100 \mu \mathrm{M})$ and PDTC $(0,3,10,30,50$ or $100 \mu \mathrm{M})$. Cells were continued to culture for 24 or $48 \mathrm{~h}$. Then, $100 \mu \mathrm{g}$ MTT $(20 \mu \mathrm{l})$ of reagent was added to each well. After $4 \mathrm{~h}$ of incubation, $150 \mu \mathrm{l}$ of DMSO was added to each well to dissolve the formed crystals under oscillation. Finally, enzyme-linked immunosorbent assay (ELISA) was performed to determine cell proliferation at $570 \mathrm{~nm}$. Groups one and two were calculated with the following formula: rate of inhibition $(\%)=(1-$ absorbance in experimental group/absorbance in control group) $\times$ $100 \%$. For group three, Jin's method was used to determine whether the two drugs had synergistic effects [21]. This $Q$ value model was also known as the probability addition method, a widely used algorithm modified from Bürgi's equation suitable for studying combinational use of traditional or herbal medicine. The equation was as follows: $\mathrm{Q}=\mathrm{EAB} /(\mathrm{EA}+\mathrm{EB}-\mathrm{EA} \times \mathrm{EB}) . \mathrm{EAB}$ represented growth inhibition of the combination group, whereas $E A$ or $E B$ represented the inhibition respectively achieved in $A(100 \mu M$ Res $)$ or $B(3,30,50$, or $100 \mu M$ PDTC). $A Q$ value between 0.85 and 1.15 indicated a simple addition, whereas $>1.15$ indicated a synergistic effect and $<0.85$ an antagonistic effect.

\section{Wound healing assay}

MG-63 cells were seeded in 6-well plates until reaching $80 \%$ confluency. Three straight wounds were introduced to each well with $200 \mu$ l pipette tips. Detached cells during the procedure were rinsed off with phosphate-buffered saline (PBS). The adherent cells were treated with $100 \mu \mathrm{M}$ Res for 24,48 or $72 \mathrm{~h}$ at $37^{\circ} \mathrm{C}$ with $5 \% \mathrm{CO}_{2}$. Microphotographs were taken at $0,24,48$ and $72 \mathrm{~h}$ to record cell migration. Image-Pro Plus 6.0 software (Cybernetics, Princeton, NJ) was employed to quantify the cell migration and calculate the migration index.

\section{Transwell migration assay}

MG-63 cells untreated or pretreated with Res $(25,50,100$ or $200 \mu \mathrm{M})$ for $48 \mathrm{~h}$ were collected by $0.25 \%$ trypsin digestion and centrifugation. Cells were re-suspended in culture medium at $5 \times 10^{5} / \mathrm{ml}$. Transwell permeable supports were purchased from Corning (NJ, USA). The upper compartments were coated with $50 \mu$ l of Matrigel $(1 \mathrm{~g} / \mathrm{l})$ at $37^{\circ} \mathrm{C}$ for $1 \mathrm{~h}$ to form artificial basement membranes. Next, $200 \mu \mathrm{l}$ of MG-63 cells were plated in the upper chambers, and $600 \mu \mathrm{l}$ of medium supplemented with $20 \%$ FBS was added to the lower compartments. Cells were cultured for $48 \mathrm{~h}$. The permeable filters were fixed with $4 \%$ paraformaldehyde. The cells from the upper surface were scraped off. The cells at the lower surface were stained with $0.05 \%$ crystal violet and counted with microscopy. The following equation was used to determine cell migration and invasion: ( 1 - invasive cells in the experimental group/invasive cells in the control group) $\times 100 \%$.

\section{TUNEL assay}

MG-63 cells were plated on coverslips in 24well plates at a density of $1 \times 10^{5} / \mathrm{ml}$ and cultured for $24 \mathrm{~h}$. Res was added to the culture medium at a final concentration of $0,25,50,100$ or $200 \mu \mathrm{M}$. After $24 \mathrm{~h}$, apoptosis in MG-63 cells was examined with the TUNEL Apoptosis Detection Kit (FITC) following the manufacturer's instructions (San Yi Biotechnology, Shanghai, China). Apoptotic cells emitted green fluorescence at $520 \pm 20 \mathrm{~nm}$, whereas the nuclear counterstain with 4',6-diamidino-2-phenylindole (DAPI) had blue fluorescence at $460 \mathrm{~nm}$. All microphotographs were taken at $20 \times$ magnification with scale bars representing $30 \mu \mathrm{m}$.

\section{Western blotting analysis}

Western blotting analysis of protein expression followed standard procedures. In brief, total proteins were isolated from MG-63 cells treated with Res $(0,25,50,100$ or $200 \mu \mathrm{M})$ for $48 \mathrm{~h}$. Protein extracts were subjected to sodium dodecyl sulfate (SDS)-polyacrylamide gel electrophoresis (PAGE) and transferred to polyvinyl difluoride (PVDF) membranes via electro-blotting. Proteins were detected with the following antibodies: phosphor (p)-Akt, Akt, Erk, p-Erk, NF-kB and caspase-3 (Cell Signaling Technology, MA, US), glyceraldehyde 3-phosphate dehydrogenase (GAPDH) and mechanistic target of rapamycin (mTOR) (Abcam, MA, USA) and goat anti-rabbit secondary antibody (Invitrogen, CA, USA). Densitometry was performed with Image J (National Institutes of Health) following web-based instructions as follows: http:// lukemiller.org/index.php/2010/11/analyzing-gelsand-western-blots-with-image-j/.

\section{Statistical analysis}

Data were processed with GraphPad Prism 5.0 (GraphPad Software, CA, USA) for figure plotting and statistical analysis. The normally distributed data were reported as mean \pm standard deviation and compared using analysis of variance (ANOVA). All experiments were triplicated. $P<0.05$ was considered statistically significant. 


\section{Results}

\section{Resveratrol inhibits osteosarcoma cell proliferation}

Res has been tested for its anti-tumor activities in a variety of in vitro and animal models, including the two recent cell culture studies in osteosarcoma $[16,18]$. To validate the results in our cell line model, we first tested the effect of Res on MG-63 osteosarcoma cell proliferation. With the MTT assay, we observed time- and dosedependent suppression of cell growth. At $24 \mathrm{~h}$, $100 \mu \mathrm{M}$ and $200 \mu \mathrm{M}$ Res had statistically significant effects compared to the untreated group (Figure $1 \mathrm{~A}$ ). The calculated IC50 for $24 \mathrm{~h}$ was 333.67 $\mu \mathrm{M}$. With prolonged exposure for $48 \mathrm{~h}$, we saw the

A

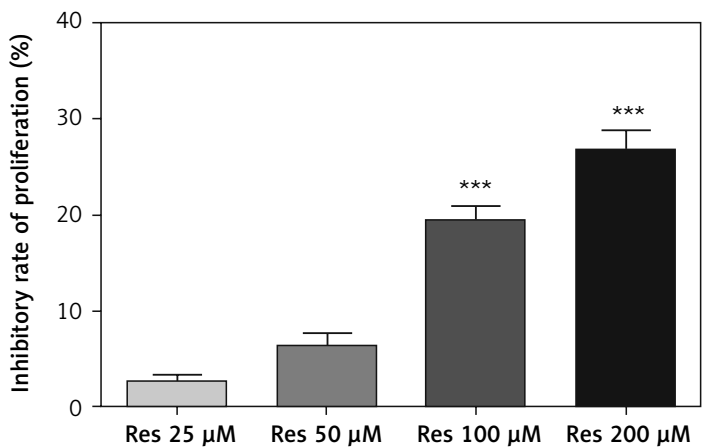

cytotoxic sensitivity extended to as low as $50 \mu \mathrm{M}$ (Figure $1 \mathrm{~B}$ ). The calculated $\mathrm{IC}_{50}$ for $48 \mathrm{~h}$ was reduced to $253.5 \mu \mathrm{M}$. This was consistent with the clinically relevant doses used by other researchers.

\section{Resveratrol suppresses osteosarcoma cell migration}

Cell migration is the key step in tumor progression from primary neoplasm to disseminated disease. We next assessed the efficacy of Res in inhibiting cell migration. We used the wound healing assay, a well-established assay of studying cell polarization, tissue remodeling, cell proliferation and migratory behaviors. Shown in Figure $2 \mathrm{~A}$, MG-63 osteosarcoma cells treated with $100 \mu \mathrm{M}$

B

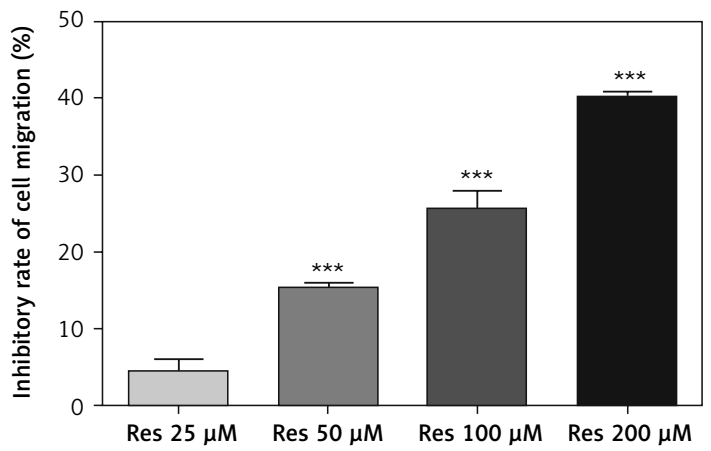

Figure 1. Resveratrol (Res) inhibits osteosarcoma MG-63 cell proliferation. A - $24 \mathrm{~h}$ of Res treatment at 100 and $200 \mu$ M significantly inhibited MG-63 cell proliferation. ${ }^{* * *} p<0.001$ compared to the untreated control, which was not plotted in the graph. B - At $48 \mathrm{~h}$, as low as $50 \mu \mathrm{M}$ Res showed significant growth inhibition of osteosarcoma MG-63 cells. ${ }^{\star * \star} p<0.001$ compared to the untreated control. All experiments were triplicated with the data expressed as mean \pm standard deviation

A

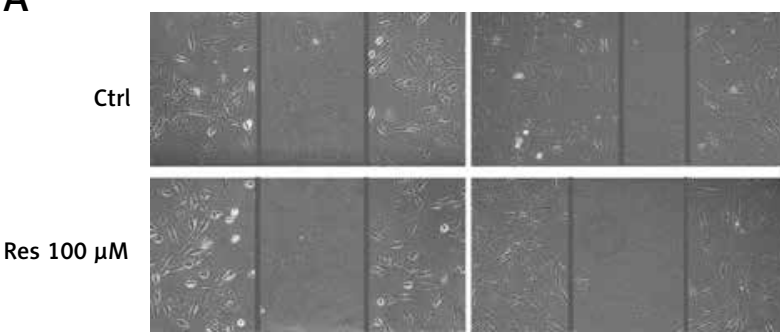

B

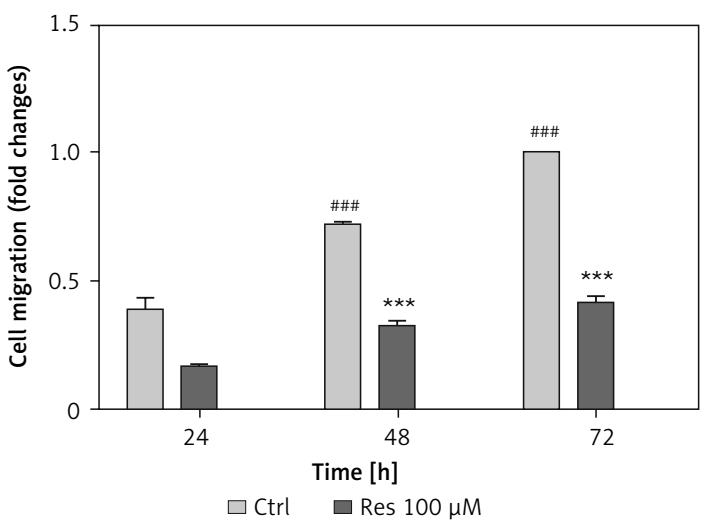

$48 \mathrm{~h}$

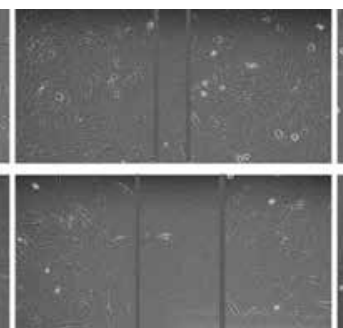

$72 \mathrm{~h}$

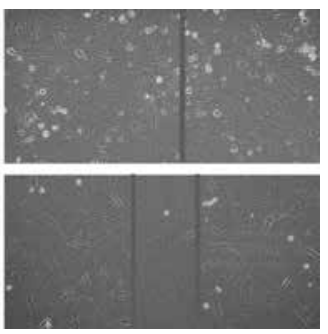

Figure 2. Res suppresses osteosarcoma MG-63 cell migration. A - Representative microphotographs $(20 x)$ of the wound healing assay. MG-63 cells were treated with $100 \mu \mathrm{M}$ Res for 24,48 or $72 \mathrm{~h}$. Solid lines represent migratory front edges. B - Quantitation of cell migration in wound healing assay. Compared to the control (ctrl), $100 \mu \mathrm{M}$ Res treatment for 48 and $72 \mathrm{~h}$ showed pronounced inhibition of osteosarcoma cell migration $\left.{ }^{* * *} p<0.001\right)$. All experiments were triplicated with the data expressed as mean \pm standard deviation 
Res displayed a temporarily diminished migratory ability and wound-healing process. This result, when quantified, showed a statistically significant difference from the untreated cells (Figure 2 B).

\section{Resveratrol reduces osteosarcoma cell invasion}

Resveratrol has been shown to hamper cell migration, with some authors suggesting that the action occurred primarily through inhibiting matrix metalloproteinase in multiple myeloma [22] and regulating Rho GTPases in breast carcinoma [23]. In osteosarcoma, a study by Yang et al. found that Res inhibited tumor migration, invasion and metastasis, possibly through the transcriptional suppression of MMP (matrix metalloproteinase)-2 via miR (microRNA)-328 [24]. With the Transwell assay, we indeed observed dose-dependent inhibition of cell invasion through the artificial basement membrane (Figure $3 \mathrm{~A}$ ). In comparison to growth inhibition (Figure 1), Res had a higher potency in suppressing cell invasion, with $25 \mu \mathrm{M}$ showing a significant difference from the untreated cells (Figure $3 \mathrm{~B}$ ).

\section{Resveratrol promotes osteosarcoma apoptotic cell death}

In earlier work [18], Res was shown to induce apoptosis in osteosarcoma cells with the Annexin $\mathrm{V}$ staining method. Annexin $\mathrm{V}$ is a calcium-dependent phospholipid-binding protein that has a high affinity for phosphatidylserine, which is translocated from the inner plasma membrane to the cell surface in apoptosis. Here we used the TUNEL assay to detect the endonucleolytic cleavage of chromatin, an event largely characteristic of late apoptosis. Shown in Figure 4, MG-63 cells treated with Res showed dose-dependent accumulation of DNA fragmentation (Figure $4 \mathrm{~A}$ ). With $24 \mathrm{~h}$ of treatment, as low as $25 \mu \mathrm{M}$ Res was capable of elucidating a strong apoptotic signal (Figure 4 B).

\section{Resveratrol regulates NF- $\kappa \mathrm{B}$ and Akt signaling in osteosarcoma}

Past studies have suggested that Res suppresses carcinogenesis by modulating intracellular signal transduction pathways. The affected signal network governs multiple crucial processes including cell division, growth, apoptosis, inflammation, angiogenesis, and metastasis. In particular to osteosarcoma, such alterations included Wnt/ $\beta$-catenin signaling and the Sirt1/Sir2 family of NAD-dependent histone deacetylases $[16,18]$. As an initial approach to probe the mechanistic basis of Res's anti-osteosarcoma activity, we subjected the total protein extracts from Res treated MG-63 cells to Western blotting analysis (Figure $5 \mathrm{~A}$ ). With caspase-3 antibody, we observed dose-dependent cleavage of the full-length caspase-3 and accumulation of the active form (quantified via densitometry, Figure 5 B). This was consistent with the observation that Res activated the canonical apoptotic pathway seen in Figure 4. Further, Res treatment resulted in dose-dependent suppression of $\mathrm{NF}-\kappa \mathrm{B}$, a key transcription complex of immune response and carcinogenesis. When used for $48 \mathrm{~h}$, as low as $25 \mu \mathrm{M}$ had a significant impact on NF- $\kappa$, when normalized to GAPDH and compared to the control (Figure $5 \mathrm{C}$ ). Akt is a serine/threonine kinase that is involved in diverse biological responses including inhibi-

A Control Res $25 \mu \mathrm{M}$ Res $50 \mu \mathrm{M}$ $\operatorname{Res} 100 \mu \mathrm{M}$ Res $200 \mu \mathrm{M}$

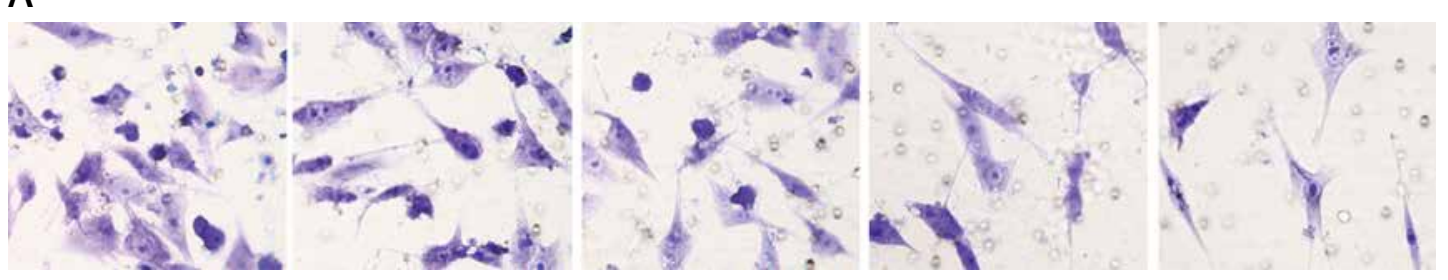

B

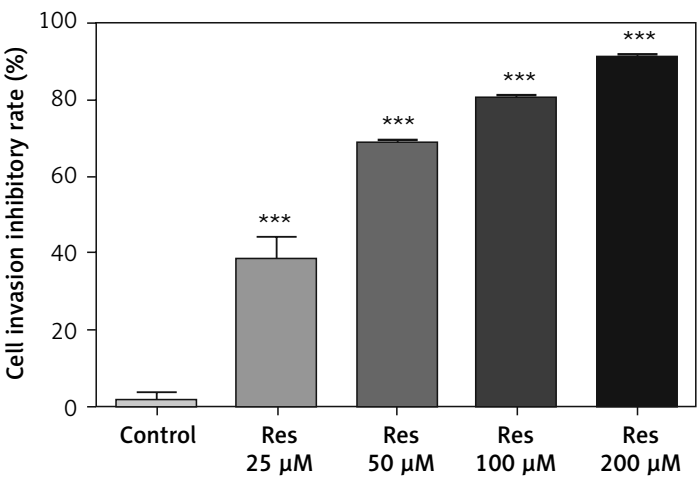

Figure 3. Res reduces osteosarcoma MG-63 cell invasion. A - Representative microphotographs (20x) of MG-63 cells in the Transwell assay. Cells were pretreated with $25,50,100$ or $200 \mu \mathrm{M}$ Res. B - Quantitation of the cell invasion in Transwell assay. Res pretreatment significantly reduced osteosarcoma cell invasion across the artificial basement membrane $\left(^{\star * *} p<0.001\right)$. All experiments were triplicated with the data expressed as mean \pm standard deviation 
A
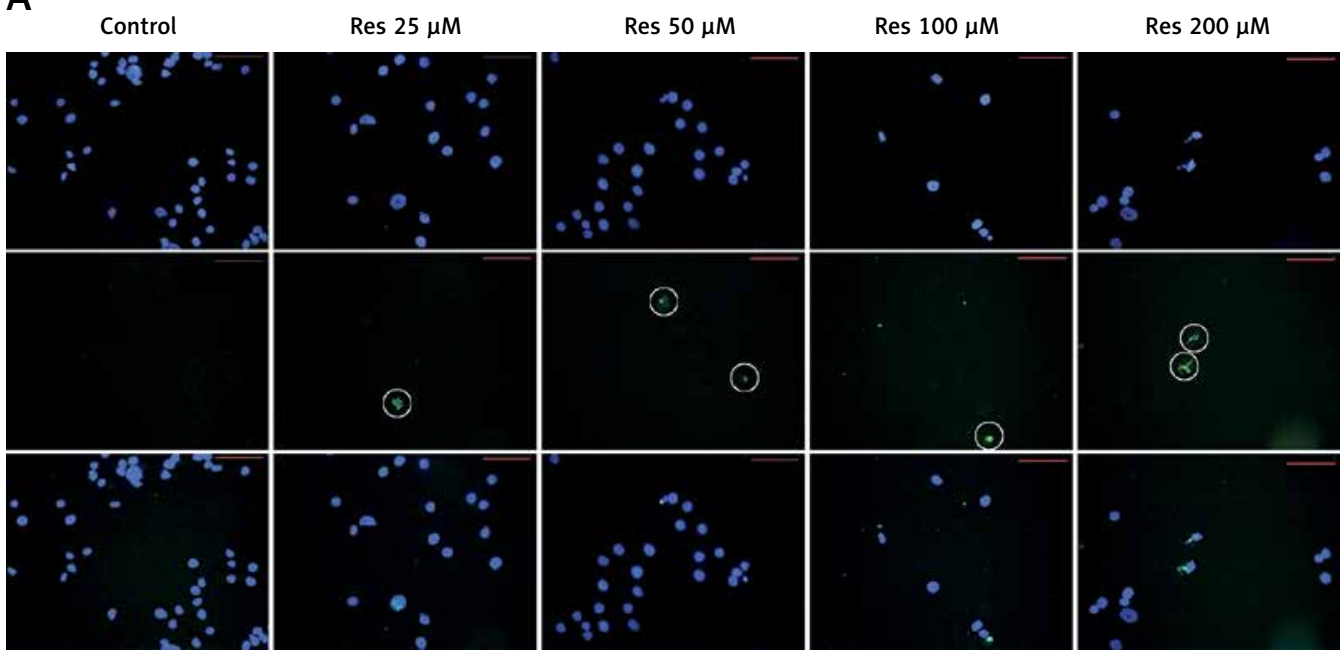

DAPI

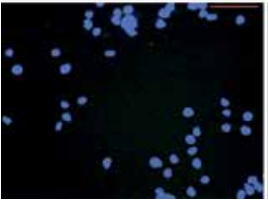

B

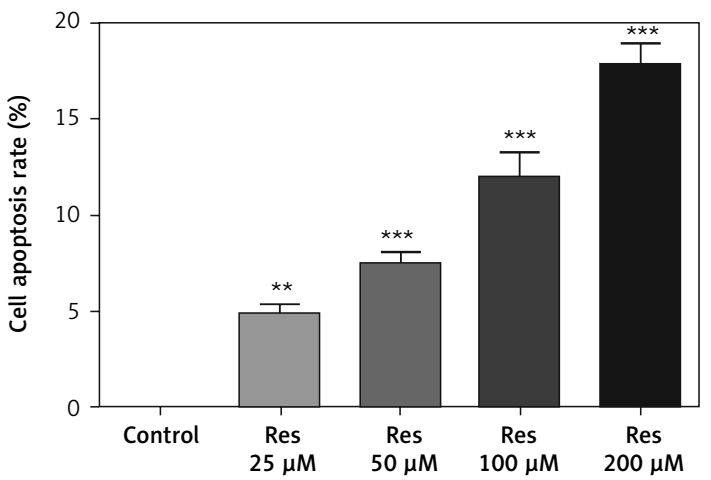

Figure 4. Res promotes MG-63 osteosarcoma apoptotic cell death. A - Representative immunofluorescence microphotographs of the TUNEL assay. MG-63 cells were treated with $25,50,100$ or $200 \mu \mathrm{M}$ Res for $24 \mathrm{~h}$. FITC detected the apoptotic cells (circled) and DAPI stained nuclei of all cells. Note the gradual decline in viable cells treated with high concentrations (100 and $200 \mu \mathrm{M})$ of Res. B - Quantitation of apoptotic cell death in TUNEL assay. Compared to the control (ctrl), Res significantly promoted cell apoptosis in osteosarcoma MG-63 cells $\left({ }^{* *} p<0.01,{ }^{* * *} p<0.001\right)$. All experiments were triplicated with the data expressed as mean \pm standard deviation. Microphotographs were taken at $20 x$ magnification with scale bars representing $30 \mu \mathrm{m}$

tion of apoptosis and stimulation of cell proliferation. Although the total Akt level responded poorly to Res treatment, the phosphor (p)-Akt showed a dose-dependent reduction, suggesting that its phosphorylation and activation were suppressed by Res (Figures 5 D, E). We also tested mechanistic target of rapamycin (mTOR), another serine/threonine kinase that has an integral role in cell growth, motility, survival, and transcription. We found that Res treatment had a neglectable effect on mTOR expression, only evident at the highest concentration, $200 \mu \mathrm{M}$ (Figure $5 \mathrm{~F}$ ). The Erk and p-Erk we tested showed a discordant response to Res treatment. At 100 and $200 \mu \mathrm{M}$ Res, total Erk showed an increase in protein expression (Figure $5 \mathrm{G}$ ), whereas the $\mathrm{p}$-Erk was modestly suppressed (Figure $5 \mathrm{H}$ ). Indeed, the effect was more pronounced when p-Erk was normalized with total Erk (results not shown). If further verified, Erk might be another downstream target of Res's pharmacological action.

\section{Synergistic effect of Res and NF- $\mathrm{KB}$} inhibitor pyrrolidine dithiocarbamate

In our results, Res treatment resulted in dose-related suppression of NF- $\mathrm{B}$, a protein complex with a recognized role in inflammation and

cancer. Chemical inhibitors of $\mathrm{NF}-\kappa \mathrm{B}$ are readily available, and many efforts have been invested to bring experimental drugs to clinical use. Our experiments suggested that NF- $\kappa B$ could be a downstream target of Res. Thus, the combination regimen of Res with an NF- $\mathrm{B} B$ inhibitor should achieve synergistic cytotoxicity. Among the list of NF- $\kappa B$ inhibitors, PDTC is well established for its role as an NF- $\kappa B$ inhibitor and antioxidant with pro-apoptotic and anti-proliferative activities across various cancer models. However, its cytotoxic profile in osteosarcoma has not been established. In this experiment, we treated MG-63 cells with increased doses of PDTC for 24 or $48 \mathrm{~h}$. At $24 \mathrm{~h}, 50$ and $100 \mu \mathrm{M}$ of PDTC showed significant inhibition of osteosarcoma proliferation in the MTT assay (Figure 6 A, IC50: $429.3 \mu \mathrm{M}$ ). Extending the treatment to $48 \mathrm{~h}$, we found that as low as $30 \mu \mathrm{M}$ of PDTC was effective in reducing cell survival (IC50: $411.4 \mu \mathrm{M}$ ). Next, we combined Res and PDTC. For Res, we used a fixed concentration of $100 \mu \mathrm{M}$, a concentration tolerated by the cells for subsequent survival analysis. For PDTC, in addition to 30 to $100 \mu \mathrm{M}$, we also included 3 and $10 \mu \mathrm{M}$. We reasoned that since PDTC is a direct NF-кB inhibitor, it may sensitize osteosarcoma to Res treatment even at below-the-threshold con- 
A
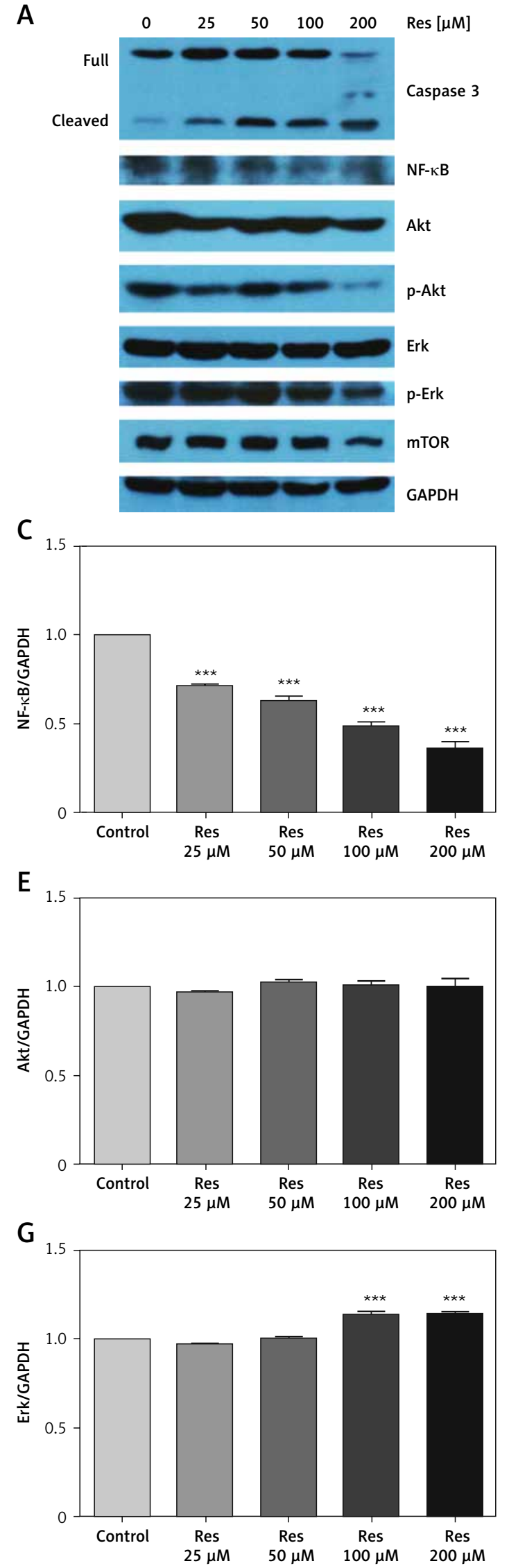

B

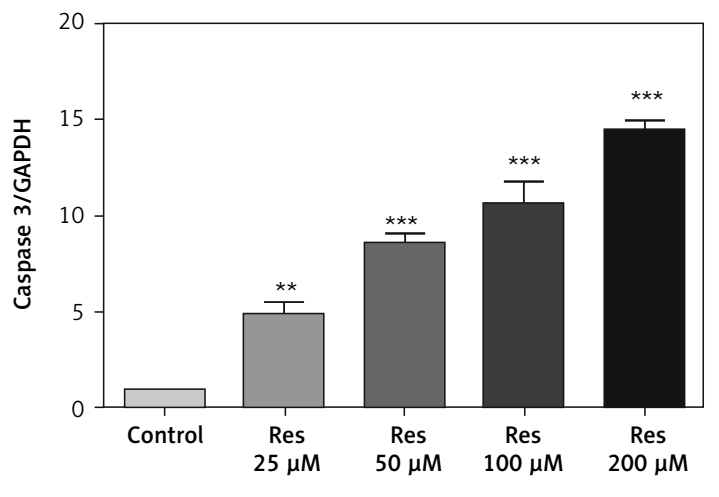

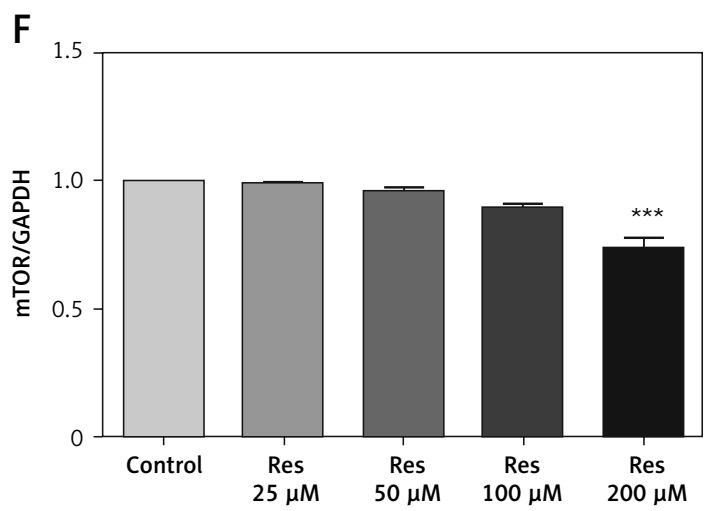

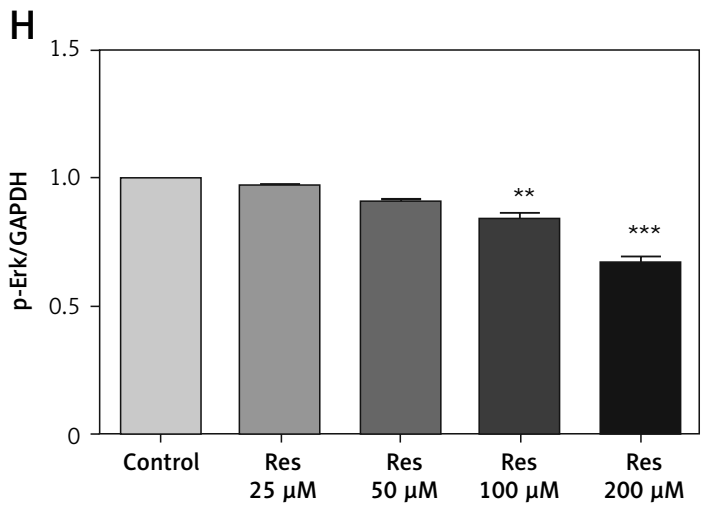

Figure 5. Res regulates Akt and NF- $\kappa B$ signaling in MG-63 osteosarcoma cells. A - Representative Western blotting analysis of various proteins affected by Res in MG-63 osteosarcoma cells. MG-63 was treated with 25 to $200 \mu \mathrm{M}$ Res for $48 \mathrm{~h}$. Glyceraldehyde 3-phosphate dehydrogenase (GAPDH) was used as loading control. B-H - Densitometric quantitation (Image J) of relative expression of cleaved caspase-3 (B), NF-kB (C), phosphor (p)-Akt (D), Akt (E), mechanistic target of rapamycin (mTOR) (F), Erk $(\mathbf{G})$ and $p$-Erk $(\mathbf{H}) .{ }^{* *} p<0.01,{ }^{* * *} p<0.001$. All experiments were triplicated with the data expressed as mean \pm standard deviation 
A

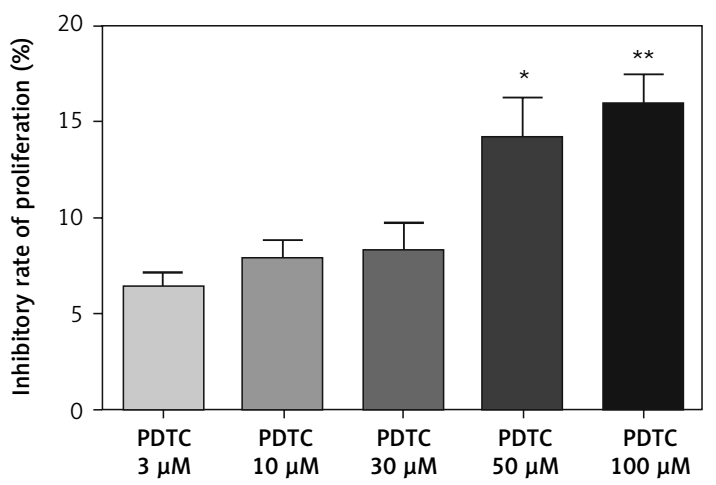

\section{B}

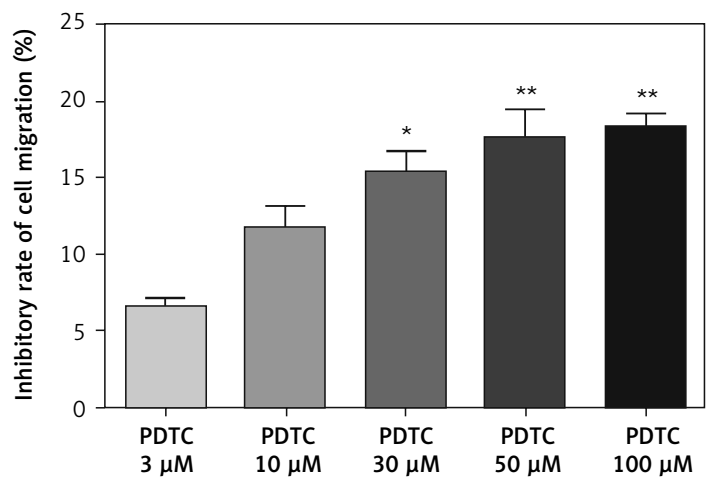

C

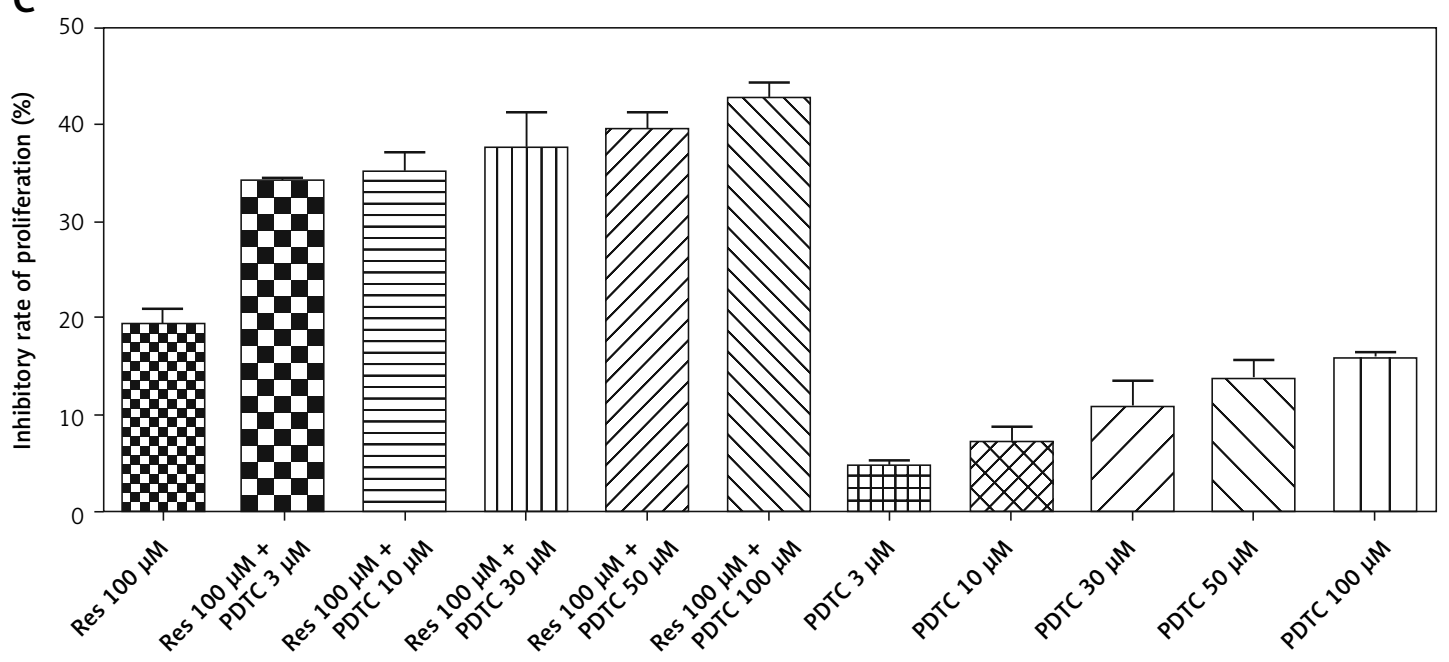

D

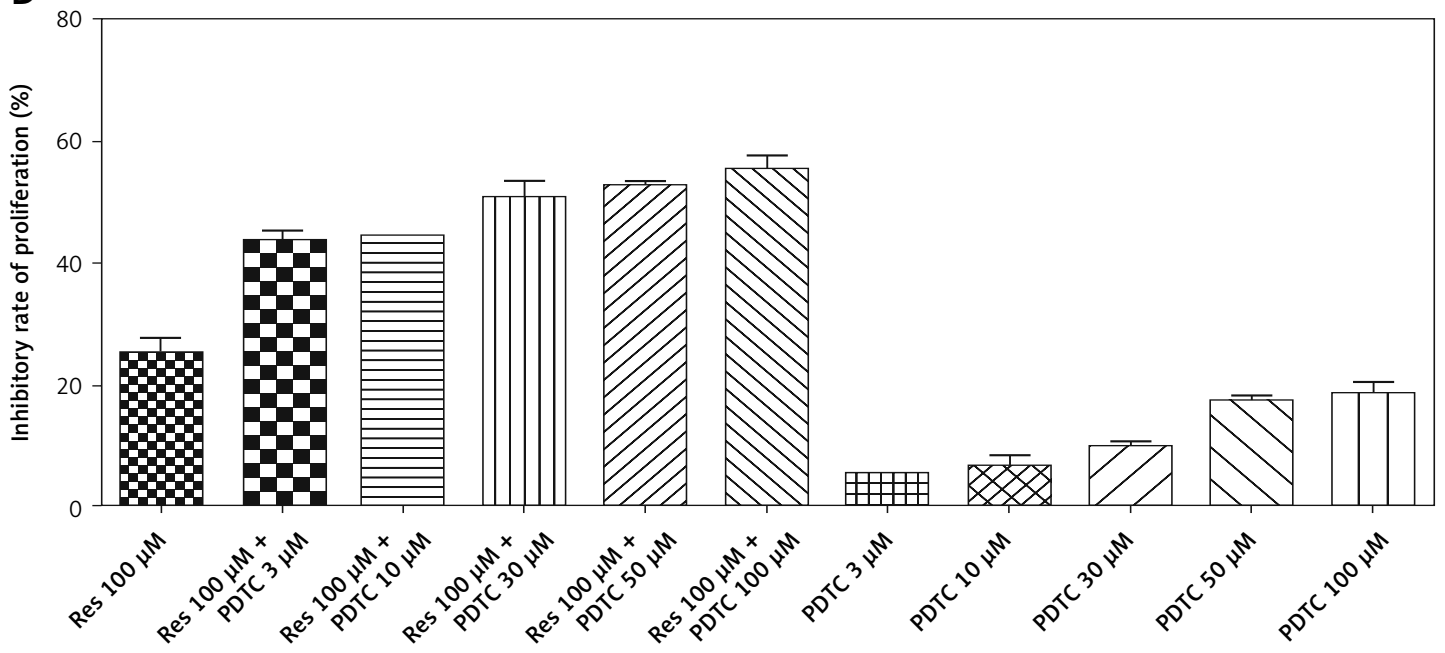

Figure 6. Synergistic effect of Res and NF- $\kappa B$ inhibitor pyrrolidine dithiocarbamate (PDTC) in suppressing MG63 osteosarcoma proliferation. A - At $24 \mathrm{~h}, 50$ and $100 \mu \mathrm{M}$ of PDTC significantly reduced MG-63 cell proliferation. The calculated IC50 for PDTC at $24 \mathrm{~h}$ was $429.3 \mu \mathrm{M}$. The calculated IC50 for PDTC at $48 \mathrm{~h}$ was $411.4 \mu \mathrm{M}$. B - At $48 \mathrm{~h}$, as low as $30 \mu \mathrm{M}$ of PDTC had a significant impact on MG-63 cell survival. For A and B: ${ }^{*} p<0.05$, ${ }^{* *} p<0.01$. All experiments were triplicated with the data expressed as mean \pm standard deviation. Combinational use of Res and PDTC in MG-63 osteosarcoma cells at 24 h (C) and 48 h (D). All experiments were triplicated with the data expressed as mean \pm standard deviation 


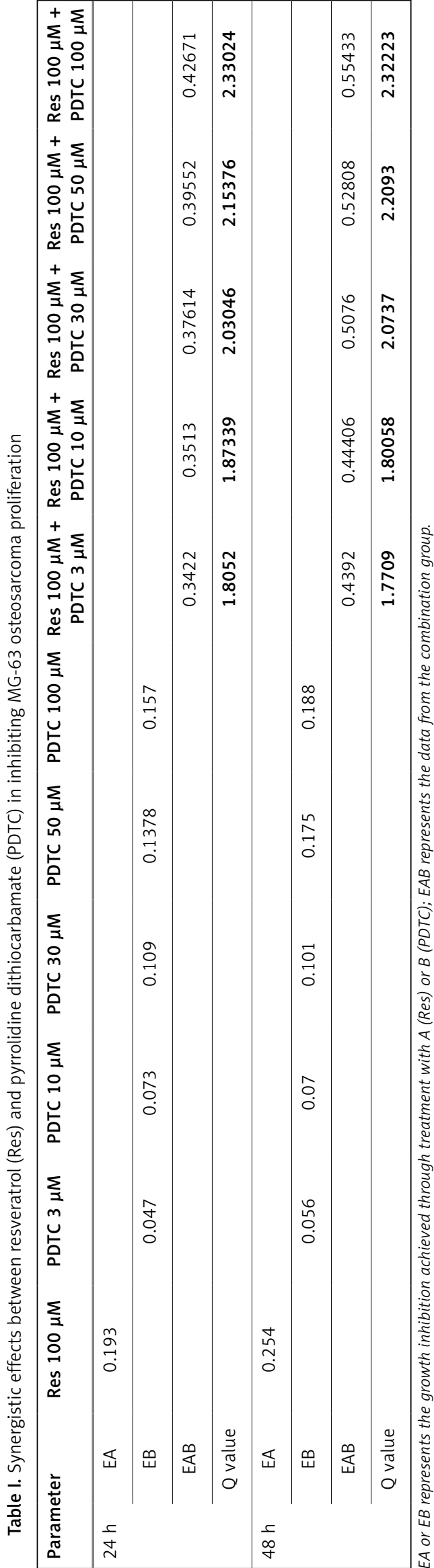

centrations. Indeed, we observed strong synergistic activity between the two drugs at all combinational doses tested for 24 or 48 h (Figures 6 C, D and Table I).

\section{Discussion}

Osteosarcoma is an aggressive primary bone tumor, leading to cancer mortality in a matter of months without clinical management. Even though there has been significant progress in the past decades, many patients failed to achieve clinically desired therapeutic outcomes. Hence, novel and more effective chemotherapies are urgently needed.

Res has been of interest lately to some researchers for its anti-tumor effects in osteosarcoma. Driven by this shared interest, we proposed the current study investigating the molecular basis of the cytotoxicity of Res in MG-63 osteosarcoma cells. We found that Res inhibited proliferation and migration, and induced apoptosis in a concentration- and/or time-dependent manner. This finding is corroborated by earlier studies [16-20, 24]. We also found that Res down-regulates the NF-kB intracellular signaling cascade. Further evidence was collected from the combination study; Res, when combined with the NF-kB inhibitor PDTC, resulted in synergistic and more pronounced growth inhibition of osteosarcoma.

NF-KB is a ubiquitously expressed gene capable of regulating a wide spectrum of target genes. The NF- $\mathrm{kB}$ signaling pathway is likely aberrantly activated in many human solid tumors, including colorectal, cervical, prostate, pancreatic, breast and thyroid cancer [25-30]. Further, NF-kB signaling also appears to intertwine with other signaling cascades such as Akt signaling. As a matter of fact, the Akt pathway was reported to converge with NF- $\mathrm{KB}$ signaling at $\mathrm{I} \mathrm{\kappa B} \alpha$ kinase (a substrate of Akt) even though these two cascades were initially considered separate [31]. Another biological link between NF-KB and PI3-kinase/AKT has also been suggested in the anti-apoptotic modulation of PEL cells [32].

In a number of past studies, Res showed significant effects on the regulation of NF-kB signaling. Kundu et al. noted that Res inhibited NF-kB activation through suppressing the phosphorylation and subsequent degradation of I $\mathrm{KB} \alpha$ in TPA-treated mouse skin [33]. Res also acted upon NF-kB by inhibiting p65 and IкB $\beta$ kinase [34]. Hence, based on the existing evidence and our present findings, we reason that NF-kB and Akt signaling pathways may be altered by Res to mediate the cytotoxicity in MG-63 cells.

Combination chemotherapies are often more efficacious in anti-tumor activity compared to the single-agent approach in cancer treatment. En- 
couraged by Res-induced regulation of NF- $\kappa B$, we examined the synergistic effect of Res and PDTC. The results indicated that combination treatment gave greater inhibition of tumor proliferation. Res has been tested in a list of combinatorial strategies (pharmaceutical or non-pharmaceutical) in preclinical models. For example, Joseph et al. suggested that Res altered the apoptotic signaling pathways when combined with caloric restriction [35]. An in vivo study investigated the combination of Res with immunotherapy in mouse neuroblastoma, where the authors noted that the combination therapy led to a significant increase in animal survival and lessened cancer metastasis [7].

Nonetheless, there are several areas of interest that worth further investigation. For one, in the current study, Res inhibited the proliferation of MG-63 cells only at concentrations exceeding $50 \mu \mathrm{M}$. The bioactivity of Res at lower doses remains unclear or less promising. A past study suggested that low-dose Res paradoxically enhanced cell survival preceding the later inhibition of cell survival [36]. In contrast, Chin and colleagues reported that low-concentration Res $(0.1 \mu \mathrm{M})$ consistently induced a decrease in cancer cell proliferation [37]. Second, the effect of Res on the Erk/p-Erk pathway opens another discussion. Our finding of inhibition of Erk phosphorylation by Res was supported by Lee et al., who investigated the antioxidant effect of Res on the fibrosarcoma cell line HT1080 [38]. On the other hand, the study by Niles et al. stated that Res activated phosphorylation of Erk in the human melanoma cells A 375 [6]. These discrepancies will have to be resolved by further studies.

In conclusion, our preclinical study demonstrates that Res inhibits cell proliferation, migration, and invasion, and activates apoptosis in the osteosarcoma MG-63 cell line model. This is likely mediated through the inhibition of NF- $\kappa B$, given that the combination of Res and NF- $\kappa$ B inhibition results in synergistic growth inhibition. Our findings thus support the translation of Res to the clinical evaluation of patients with osteosarcoma.

\section{Conflict of interest}

The authors declare no conflict of interest.

\section{References}

1. Mirabello L, Troisi RJ, Savage SA. Osteosarcoma incidence and survival rates from 1973 to 2004: data from the surveillance, epidemiology, and end results program. Cancer 2009; 115: 1531-43.

2. Torigoe T, Yazawa Y, Takagi T, Terakado A, Kurosawa H. Extraskeletal osteosarcoma in Japan: multiinstitutional study of 20 patients from the Japanese Musculoskeletal Oncology Group. J Orthop Sci 2007; 12: 424-9.
3. Boon E, van der Graaf WT, Gelderblom H, et al. Impact of chemotherapy on the outcome of osteosarcoma of the head and neck in adults. Head Neck 2017; 39: 140-6.

4. Smith MA, Altekruse SF, Adamson PC, Reaman GH, Seibel NL. Declining childhood and adolescent cancer mortality. Cancer 2014; 120: 2497-506.

5. Luetke A, Meyers PA, Lewis I, Juergens H. Osteosarcoma treatment - where do we stand? A state of the art review. Cancer Treat Rev 2014; 40: 523-32.

6. Niles RM, McFarland M, Weimer MB, Redkar A, Fu YM, Meadows GG. Resveratrol is a potent inducer of apoptosis in human melanoma cells. Cancer Lett 2003; 190: 157-63.

7. Soto BL, Hank JA, Van De Voort TJ, et al. The anti-tumor effect of resveratrol alone or in combination with immunotherapy in a neuroblastoma model. Cancer Immunol Immunother 2011; 60: 731-8.

8. Fraser SP, Peters A, Fleming-Jones S, Mukhey D, Djamgoz MB. Resveratrol: inhibitory effects on metastatic cell behaviors and voltage-gated $\mathrm{Na}(+)$ channel activity in rat prostate cancer in vitro. Nutr Cancer 2014; 66: 1047-58.

9. Mitani T, Ito Y, Harada N, et al. Resveratrol reduces the hypoxia-induced resistance to doxorubicin in breast cancer cells. J Nutr Sci Vitaminol 2014; 60: 122-8.

10. Jang M, Cai L, Udeani GO, et al. Cancer chemopreventive activity of resveratrol, a natural product derived from grapes. Science 1997; 275: 218-20.

11. Buhrmann C, Shayan P, Popper B, Goel A, Shakibaei M. Sirt1 is required for resveratrol-mediated chemopreventive effects in colorectal cancer cells. Nutrients 2016; 8: 145

12. Kato A, Naiki-Ito A, Nakazawa T, et al. Chemopreventive effect of resveratrol and apocynin on pancreatic carcinogenesis via modulation of nuclear phosphorylated GSK3beta and ERK1/2. Oncotarget 2015; 6: 42963-75.

13. Sinha D, Sarkar N, Biswas J, Bishayee A. Resveratrol for breast cancer prevention and therapy: preclinical evidence and molecular mechanisms. Semin Cancer Biol 2016; 40-41: 209-32.

14. Billard C, Izard JC, Roman V, et al. Comparative antiproliferative and apoptotic effects of resveratrol, epsilon-viniferin and vine-shots derived polyphenols (vineatrols) on chronic B lymphocytic leukemia cells and normal human lymphocytes. Leuk Lymphoma 2002; 43: 1991-2002.

15. Zulueta A, Caretti A, Signorelli P, Ghidoni R. Resveratrol: a potential challenger against gastric cancer. World J Gastroenterol 2015; 21: 10636-43.

16. Zou Y, Yang J, Jiang D. Resveratrol inhibits canonical Wnt signaling in human MG-63 osteosarcoma cells. Mol Med Rep 2015; 12: 7221-6.

17. Sun Y, Wang H, Liu M, Lin F, Hua J. Resveratrol abrogates the effects of hypoxia on cell proliferation, invasion and EMT in osteosarcoma cells through downregulation of the HIF-1alpha protein. Mol Med Rep 2015; 11: 1975-81.

18. Li Y, Bäckesjö CM, Haldosén LA, Lindgren U. Resveratrol inhibits proliferation and promotes apoptosis of osteosarcoma cells. Eur J Pharmacol 2009; 609: 13-8.

19. Liu Y, Wang X, Xie Y, Zhang J, Wang Q, Xu X. Resveratrol induces apoptosis in human osteosarcoma MG63 cells. Chin J Clin Oncol 2008; 5: 361.

20. Liu Z, Li Y, Yang R. Effects of resveratrol on vascular endothelial growth factor expression in osteosarcoma cells and cell proliferation. Oncol Lett 2012; 4: 837-9.

21. Jin Z. Synergy in drug combination. Acta Pharmacol Sin 1980; 1: 70-3.

22. Sun CY, Hu Y, Guo T, et al. Resveratrol as a novel agent for treatment of multiple myeloma with matrix metallo- 
proteinase inhibitory activity. Acta Pharmacol Sin 2006; 27: $1447-52$

23. Azios NG, Krishnamoorthy L, Harris M, Cubano LA, Cammer M, Dharmawardhane SF. Estrogen and resveratrol regulate Rac and Cdc42 signaling to the actin cytoskeleton of metastatic breast cancer cells. Neoplasia 2007; 9: 147-58.

24. Yang SF, Lee WJ, Tan P, et al. Upregulation of miR-328 and inhibition of CREB-DNA-binding activity are critical for resveratrol-mediated suppression of matrix metalloproteinase- 2 and subsequent metastatic ability in human osteosarcomas. Oncotarget 2015; 6: 2736-53.

25. Myant KB, Cammareri P, McGhee EJ, et al. ROS production and NF-kappaB activation triggered by RAC1 facilitate WNT-driven intestinal stem cell proliferation and colorectal cancer initiation. Cell Stem Cell 2013; 12: 761-73.

26. Branca M, Giorgi C, Ciotti $M$, et al. Upregulation of nuclear factor-kappaB (NF-kappaB) is related to the grade of cervical intraepithelial neoplasia, but is not an independent predictor of high-risk human papillomavirus or disease outcome in cervical cancer. Diagn Cytopathol 2006; 34: 555-63.

27. Park $\mathrm{MH}$, Choi MS, Kwak DH, et al. Anti-cancer effect of bee venom in prostate cancer cells through activation of caspase pathway via inactivation of NF-kappaB. Prostate 2011; 71: 801-12.

28. Maniati E, Bossard M, Cook N, et al. Crosstalk between the canonical NF-kappaB and Notch signaling pathways inhibits PPARgamma expression and promotes pancreatic cancer progression in mice. J Clin Invest 2011; 121: 4685-99.

29. Schlotter CM, Vogt U, Allgayer H, Brandt B. Molecular targeted therapies for breast cancer treatment. Breast Cancer Res 2008; 10: 211.

30. Meng Z, Mitsutake N, Nakashima M, et al. Dehydroxymethylepoxyquinomicin, a novel nuclear factorkappaB inhibitor, enhances antitumor activity of taxanes in anaplastic thyroid cancer cells. Endocrinology 2008; 149: 5357-65.

31. Sizemore N, Leung S, Stark GR. Activation of phosphatidylinositol 3-kinase in response to interleukin-1 leads to phosphorylation and activation of the NF-kappaB p65/RelA subunit. Mol Cell Biol 1999; 19: 4798-805.

32. Hussain AR, Ahmed SO, Ahmed M, et al. Cross-talk between NFkB and the PI3-kinase/AKT pathway can be targeted in primary effusion lymphoma (PEL) cell lines for efficient apoptosis. PLoS One 2012; 7: e39945.

33. Kundu JK, Shin YK, Kim SH, Surh YJ. Resveratrol inhibits phorbol ester-induced expression of COX-2 and activation of NF-kappaB in mouse skin by blocking IkappaB kinase activity. Carcinogenesis 2006; 27: 1465-74.

34. Ren Z, Wang L, Cui J, et al. Resveratrol inhibits NF-kappaB signaling through suppression of p65 and IkappaB kinase activities. Pharmazie 2013; 68: 689-94.

35. Joseph AM, Malamo AG, Silvestre J, et al. Short-term caloric restriction, resveratrol, or combined treatment regimens initiated in late-life alter mitochondrial protein expression profiles in a fiber-type specific manner in aged animals. Exp Gerontol 2013; 48: 858-68.

36. Narayanan BA, Narayanan NK, Re GG, Nixon DW. Differential expression of genes induced by resveratrol in LNCaP cells: P53-mediated molecular targets. Int J Cancer 2003; 104: 204-12.

37. Chin YT, Hsieh MT, Yang SH, et al. Anti-proliferative and gene expression actions of resveratrol in breast cancer cells in vitro. Oncotarget 2014; 5: 12891-907.
38. Lee SJ, Kim MM. Resveratrol with antioxidant activity inhibits matrix metalloproteinase via modulation of SIRT1 in human fibrosarcoma cells. Life Sci 2011; 88: 465-72. 